\title{
Maresin 1 promotes nerve regeneration and alleviates neuropathic pain after nerve injury
}

\section{Jinhuan Wei}

Nantong University https://orcid.org/0000-0003-2887-1564

Gang Chen ( $\nabla$ chengang6626@ntu.edu.cn )

Nantong University https://orcid.org/0000-0003-3669-5687

\section{Wenfeng Su}

Nantong University

\section{Yayu Zhao}

Nantong University

\section{Zhongya Wei}

Nantong University

\section{Yuchen Hua}

Nantong University

\section{Peng Xue}

Nantong University

\section{Xiang Zhu}

Nantong University

\section{Ying Chen}

Nantong University

\section{Research}

Keywords: Maresin 1, inflammation, nerve regeneration, neurological recovery, neuropathic pain, never injury

Posted Date: May 5th, 2021

DOl: https://doi.org/10.21203/rs.3.rs-448859/v1

License: (1) This work is licensed under a Creative Commons Attribution 4.0 International License. Read Full License

Version of Record: A version of this preprint was published at Journal of Neuroinflammation on February 2nd, 2022. See the published version at https://doi.org/10.1186/s12974-022-02405-1. 


\section{Abstract}

Background: Peripheral nerve injury (PNI) is a public health concern that results in sensory and motor disorders as well as neuropathic pain and secondary lesions. Currently, effective treatments for PNI are still limited. For example, while nerve growth factor (NGF) is widely used in the treatment of PNI to promote nerve regeneration, it also induces pain. Maresin 1 (MaR1) is an anti-inflammatory and proresolving mediator that has the potential to regenerate tissue. We determined whether MaR1 is able to promote nerve regeneration as well as alleviating neuropathic pain, and to be considered as a putative therapeutic agent for treating PNI.

Methods: PNI models were constructed with eight-week-old adult male ICR mice and treated with NGF, MaR1 or saline by local application, intrathecal injection or intraplantar injection. Behavioral analysis and muscle atrophy test were assessed after treatment. Immunofluorescence assay was performed to examine the expression of ATF-3, GFAP, IBA1, and NF200. The expression transcript levels of inflammatory factors IL1 $\beta$, IL- 6 , and TNF-a were detected by quantitative real-time RT-PCR. AKT, ERK, mTOR, PI3K, phosphorylated AKT, phosphorylated ERK, phosphorylated mTOR, and phosphorylated PI3K levels were examined by western blot analysis. Whole-cell patch-clamp recordings were executed to detect transient receptor potential vanilloid 1 (TRPV1) currents.

Results: MaR1 demonstrated a more robust ability to promote sensory and motor function recovery in mice after sciatic nerve crush injury than NGF. Immunohistochemistry analyses showed that the administration of MaR1 to mice with nerve crush injury reduced the number of damaged DRG neurons, promoted injured nerve regeneration and inhibited gastrocnemius muscle atrophy. Western blot analysis of ND7/23 cells cultured with MaR1 revealed that MaR1 regulated neurite outgrowth through the PI3KAKT-mTOR signaling pathway. Moreover, MaR1 dose-dependently attenuated the mechanical allodynia and thermal hyperalgesia induced by nerve injury. Consistent with the analgesic effect, MaR1 inhibited capsaicin-elicited TRPV1 currents, repressed the nerve injury-induced activation of spinal microglia and astrocytes and reduced the production of proinflammatory cytokines in the spinal cord dorsal horn in PNI mice.

Conclusions: Application of MaR1 to PNI mice significantly promoted nerve regeneration and alleviated neuropathic pain, suggesting that MaR1 is a promising therapeutic agent for PNI.

\section{Background}

Peripheral nerve injury (PNI) is a common clinical disease that is usually caused by sudden crushing, strong external force, ischemic injuries, transection, or other iatrogenic injuries. Patients with PNI are somewhat prohibited by sensory and motor disorders and endure neuropathic pain and its secondary lesions. Various experimental and clinical strategies, such as nerve grafts, nerve transfers, nerve conduits, cell-based therapies, and gene therapy, have been implemented to improve neuron survival, improve axon regeneration and target reinnervation $[1,2]$. The use of appropriate cytokines to protect damaged neurons 
and promote axonal regeneration has always been an important strategy in the study of nerve regeneration. Among cytokines, nerve growth factor (NGF) is well known to play key roles in neuronal survival, growth and maintenance in response to injury [3]. However, researchers studying the effects of NGF on PNI have not paid much attention to its side effect of inducing neuropathic pain [4]. For the last two decades, an increasing number of studies have focused on anti-NGF therapy [5]. Since NGF plays two roles in the treatment of $\mathrm{PNI}$, improving nerve regeneration but accelerating neuropathic pain, the discovery of novel cytokine(s) or drug molecular(s) that can protect injured neurons, promote axonal regeneration, and inhibit neuropathic pain in the treatment of PNI is a very urgent and important task.

Maresin 1 (MaR1), an anti-inflammatory and proresolving mediator, is a dioxygenation product produced by human macrophages that was discovered and named by Serhan et al. [6]. It has shown promising value in the treatment of airway inflammation, pneumonia, colitis, delayed wound healing and diabetes complications and has been shown to ameliorate pain hypersensitivity and provide neuronal protection [7-11]. In planaria, MaR1 promoted the speed of head reappearance and increased the rate of surgical regeneration [12]; in rats and mice, MaR1 promoted bone regeneration [13, 14]. These results prompted us to investigate whether MaR1 can simultaneously exert anti-inflammatory and analgesic effects and accelerate nerve regeneration after nervous system injury.

In this report, we examined whether MaR1 could promote nerve regeneration and neurological functional recovery and alleviate neuropathic pain after nerve injury and compared its effects with those of NGF. Our study demonstrates that MaR1 is a promising PNI therapeutic agent due to its dual functions of improving neural regeneration and protecting injured neurons while prohibiting neuropathic pain by inhibiting glial cell activation and the inflammatory response in the spinal cord.

\section{Methods}

\section{Mice and surgery}

Mouse experiments were performed according to guidelines established by the Institutional Animal Care and Use Committee of Nantong University. The ICR mice used for the experiments were obtained from the Laboratory Animal Center of Nantong University. Primary dorsal root ganglion (DRG) neurons were isolated from newborn ICR mice (postnatal day 0-1). Eight-week-old adult male ICR mice (25-30 g) were used to construct the PNI models, including a sciatic nerve crush model and a sciatic nerve chronic constriction injury model (CCl). The sciatic nerve crush injury model was established according to our previous report [15]. Briefly, after anaesthetization with isoflurane, the left sciatic nerve of the mouse was squeezed with no. 5 jeweler's forceps for 20 seconds. In some cases, a diluted fluorescent dye $(0.5 \mu \mathrm{l}$ of Vybrant CM-Dil in $20 \mu$ l of PBS) was injected into the hind paw on the injured side at 7 days after nerve crushing, and L5 DRG sections were examined seven days later. The $\mathrm{CCI}$ model was produced by placing three ligatures (7-0 Prolene, $1 \mathrm{~mm}$ intervals) around the left sciatic nerve proximal to the trifurcation. The ligatures were softly tied until a short flick of the ipsilateral hind limb occurred [16]. The mice in the sham group were subjected to the surgery described above but not to nerve injury. The mice were separated into 
groups (5 mice per cage) and housed under standard conditions $\left(25 \pm 1^{\circ} \mathrm{C}, 12-\mathrm{h}\right.$ light/dark cycle, ad libitum access to food and water).

\section{Reagents and administration}

Capsaicin (catalog: 404-86-4) and NGF (catalog: 01-170) were purchased from Sigma-Aldrich. Maresin 1 (7R,14S-dihydroxy-docosa-4Z,8E,10E,12Z,16Z,19Z- hexaenoic acid) was purchased from Cayman Chemical Company (catalog: 1268720-28-0). Vybrant CM-Dil Cell-Labeling Solution was purchased from Invitrogen (catalog: V22885). A sterilized hemostatic gelatin sponge containing $500 \mathrm{ng}$ of MaR1 or saline (control) was immediately applied locally to the injured nerve after crushing, and the wound was then closed. Drugs in $20 \mu \mathrm{l}$ of PBS were intraplantarly injected using a Hamilton microsyringe with a $30-\mathrm{G}$ needle. The spinal cord was punctured with a 30-gauge needle between the L5 and L6 levels for intrathecal drug delivery.

\section{Cell culture}

DRG neurons were harvested from newborn ICR mice and then subjected to explant culture or dissociated culture as previously described [17]. In brief, ND7/23 rat DRG/mouse neuroblastoma hybrid cells were obtained from Sigma-Aldrich and maintained in DMEM supplemented with 10\% FBS, 2 mM L-glutamate and $10 \%$ penicillin/streptomycin.

\section{Whole-cell patch-clamp recordings in dissociated mouse DRG neurons}

As we described previously [18], whole-cell patch-clamp recordings in dissociated DRG neurons (small size, $<25 \mathrm{~mm}$ ) harvested from 4- to 6-week-old mice were performed at room temperature using an Axopatch-200B amplifier (Axon Instruments, USA). The patch pipettes were pulled from borosilicate capillaries (Chase Scientific Glass Inc., Rockwood, TN, USA), and their resistance when filled with the pipette solution (in mM: $126 \mathrm{~K}$-gluconate, $10 \mathrm{NaCl}, 1 \mathrm{MgCl}_{2}, 10 \mathrm{EGTA}, 2 \mathrm{NaATP}$, and $0.1 \mathrm{MgGTP}$, adjusted to $\mathrm{pH} 7.3$ with $\mathrm{KOH}$ ) was 4-5 $\mathrm{M} \Omega$. Whole-cell recordings were performed in an extracellular solution (in $\mathrm{mM}$ ): $140 \mathrm{NaCl}, 5 \mathrm{KCl}, 2 \mathrm{CaCl}_{2}, 1 \mathrm{MgCl}_{2}, 10 \mathrm{HEPES}, 10$ glucose, adjusted to $\mathrm{pH} 7.4 \mathrm{NaOH}$. A voltage clamp was applied at a holding membrane potential of $-70 \mathrm{mV}$ to record the inward currents, and the recording chamber $(300 \mu \mathrm{l})$ was superfused continuously $(3-4 \mathrm{~mL} / \mathrm{min})$. We compensated for series resistance $(>80 \%)$ and performed leak subtraction. The data were low-pass filtered at $2 \mathrm{kHz}$ and sampled at $10 \mathrm{kHz}$, and pClamp10 (Axon Instruments) software was used for the experiments and data analysis.

\section{Behavioral analysis}

A total of four double-blind behavioral tests were used. 1) Walking track (footprint) analysis was used to analyze basic motor functions, and the sciatic function index (SFI) was calculated as previously reported [15]. In brief, the plantar surface of each mouse's hind paw was smeared with ink, and the mouse was allowed to walk in a straight path on white paper. 2) The rotarod test was used to test complex motor functions as previously reported [19]. Briefly, mice were tested three times at 10-minute intervals, and the 
time spent on the rod was recorded and averaged. During the test, the speed was increased from 2 to 20 rpm over a three-minute period. 3) The von Frey test was used to test mechanical sensitivity as previously reported [18]. Briefly, the mice were placed in a box on an elevated metal mesh floor and stimulated with a series of von Frey filaments of logarithmically increasing stiffness (0.02-2.56 gf; Stoelting) on their hind paws. The $50 \%$ paw withdrawal threshold was determined by Dixon's up-down method. 4) Using a Hargreaves radiant heat apparatus (IITC Life Science) as previously reported [19], we tested the thermal sensitivity. The basal paw withdrawal latency was adjusted to 9 to 12 seconds with a cutoff of 20 seconds to avoid tissue damage.

\section{Immunofluorescence assay}

As we described previously [20], the mice were deeply anesthetized with isoflurane, and their ascending aortas were perfused first with PBS and then with 4\% paraformaldehyde. After perfusion, the L4-L6 spinal cord segments were collected and then postfixed overnight. The spinal cord sections were sliced at a thickness of $30 \mu \mathrm{m}$ (free-floating) on a cryostat and processed for immunohistochemistry analysis. The sections were first blocked with $2 \%$ goat serum at room temperature for one hour and then incubated at $4^{\circ} \mathrm{C}$ overnight with the following primary antibodies: anti-ATF-3 (rabbit, 1:1000, Santa Cruz Biotechnology Inc.), anti-GFAP (mouse, 1:1000, EMD Millipore), anti-IBA1 (rabbit, 1:1000; Wako Chemicals Inc., USA) and anti-NF200 (mouse, 1:1,000, Sigma, catalog: N0142). After washing, the sections were incubated at room temperature for two hours with the following secondary antibodies (1:400, Jackson ImmunoResearch): Cy3-donkey anti-rabbit (catalog: 711-165-152) and FITC-donkey anti-mouse (catalog: 715-095-150). The stained sections were observed and photographed with a Leica fluorescence microscope.

\section{Muscle atrophy test}

After the administration of MaR1 for nine days after $\mathrm{CCl}$, the gastrocnemius muscles from both hind legs were separated for imaging and weight measurements. The muscle size and weight were used to assess muscle atrophy.

\section{Quantitative real-time RT-PCR}

Total RNA was collected from ipsilateral and contralateral spinal dorsal horn tissues using TRIzol reagent (Life Technologies, USA), and $1 \mu \mathrm{g}$ of RNA was reverse-transcribed using the PrimeScript RT reagent kit (Takara, Dalian, China). Gene-specific mRNA analyses were performed using the MiniOpticon Real-Time PCR system (BioRad), and the mRNA expression levels were calculated using the 2- $\Delta \Delta \mathrm{Ct}$ method. The specific primers, including those for the GAPDH control, were synthesized by Thermo Fisher Scientific, and the sequences were as follows: IL1 $\beta$ (forward TACATCAGCACCTCACAAGC, reverse AGAAACAGTCCAGCCCATACT), IL-6 (forward TCCATCCAGTTGCCTTCTTGG, reverse CCACGATTTCCCAGAGAACATG), TNF-a (forward CCCCAAAGGGATGAGAAGTT, reverse CACTTGGTGGTTTGCTACGA) and GAPDH (forward TTGATGGCAACAATCTCCAC, reverse CGTCCCGTAGACAAAATGGT). 


\section{Western blot analysis}

Total proteins were extracted from ND7/23 cells with RIPA lysis buffer (Beyotime, Shanghai, China) containing a protease inhibitor cocktail and phosphate inhibitors (Roche Molecular Biochemicals, Inc. Mannheim, Germany). The proteins were separated on $8 \%$ or $10 \%$ SDS-PAGE gels and electrophoretically transferred onto PVDF membranes. The membranes were blocked with $5 \%$ nonfat milk for $1-2 \mathrm{~h}$ and then incubated with antibodies against phosphorylated AKT (p-AKT) (1:1000, rabbit, Cell Signaling, catalog: 9271), AKT (1:1000, rabbit, Cell Signaling, catalog: 9272), phosphorylated ERK (p-ERK) (1:1000, rabbit, Cell Signaling, catalog: 9101), total ERK (1:1000, rabbit, Cell Signaling, catalog: 9102), phosphorylated mTOR (p-mTOR) (1:1000, rabbit, Cell Signaling, catalog: 2971), mTOR (1:1000, rabbit, Cell Signaling, catalog: 2972), phosphorylated PI3K (p-PI3K) (1:1000, rabbit, catalog: 4228), PI3K (1:1000, rabbit, Cell Signaling, catalog: 4292), and GAPDH (1:10000, mouse, Proteintech, catalog: 60004-1-lg) overnight at $4^{\circ} \mathrm{C}$. The next day, the membranes were incubated with the corresponding secondary antibody at room temperature for $2 \mathrm{~h}$. Bands were detected using PierceTM ECL western blotting substrate (Thermo, USA), and the results were analyzed by ImageJ software.

\section{Statistical analyses}

All data are expressed as the mean \pm SEM, as indicated in the figure captions. Student's t-test (two groups) or ANOVA (one-way and two-way) was used to compare the differences between groups, followed by Bonferroni's test. The criterion for statistical significance was $P<0.05$.

\section{Results}

\section{Sciatic nerve crush injury caused motor dysfunction and neuropathic pain in mice}

We aimed to study both nerve regeneration and neuropathic pain in one model. Therefore, we established a sciatic nerve crush injury model in mice and examined motor and sensory functions and pain behavior after nerve injury. We first used the SFI evaluation to assess the motor function recovery of the affected hindlimb. The footprint test showed that the mice developed severe motor dysfunction at 3 days after sciatic nerve injury, and the recovery took almost 4 weeks (Figure 1A). Next, we performed a rotarod test to evaluate the fine motor functions of the mice after nerve crushing. Severely impaired fine motor functions were observed after nerve injury, and recovery took 12 weeks (Figure 1B). This result differed from that of the footprint test, which showed a full recovery of basic motor function after 4 weeks. Moreover, we investigated pain behavior by the von Frey and Hargreaves tests. In the first week, the mice with nerve injury showed a higher mechanical withdrawal threshold than normal mice, and pain sensitivity was high during the initial recovery period, peaking in the third week but lasting for more than 12 weeks (Figure 1C). The Hargreaves test results showed that the paw withdrawal latencies of nerveinjured mice recovered to normal levels after 11 weeks (Figure 1D). Therefore, in this model, both motor and sensory functions were damaged to some degree, and pain behaviors were obvious.

\section{MaR1 stimulated axon regeneration}


A previous study reported that MaR1 potentially stimulated tissue regeneration in planaria [12]. and we herein investigated whether MaR1 could promote nerve regeneration in injured mice. To answer this question, we harvested newborn mouse DRGs and then cultured them with MaR1. After the administration of MaR1 $(10 \mathrm{ng} / \mathrm{ml})$ for 5 days, the DRG explants showed more extending nerve fiber bundles and more neurons than those not treated with MaR1 (Figure 2A). Similar results were observed when MaR1 was applied to dissociated DRG cell cultures for $36 \mathrm{~h}$. The DRGs in the MaR1 group displayed substantially more and longer neurites than those in the control group without MaR1 treatment (Figure 2B). Therefore, MaR1 directly influences neurite outgrowth in DRG cells from both dissociated and explant cultures.

Most neurotrophic factors promote neurite outgrowth in a dose-dependent manner [21]. To determine the optimal dosage of MaR1 for further study, we cultured DRG cells with the agent at a series of concentrations $(0.1,0.5,1,3,10,100 \mathrm{ng} / \mathrm{ml})$ for $36 \mathrm{~h}$. MaR1 $(1,3,10,100 \mathrm{ng} / \mathrm{ml})$ significantly increased the length and number of neurites (Figure $2 \mathrm{C}$ and $2 \mathrm{D}, \mathrm{P}<0.05$ ) in a dose-dependent manner compared with those in the control group. However, the results of the $100 \mathrm{ng} / \mathrm{ml} \mathrm{MaR} 1 \mathrm{group}$ were not significantly different from those of the $10 \mathrm{ng} / \mathrm{ml} \mathrm{MaR} 1$ group. In addition, we assessed the EC50 values (concentration needed to achieve $50 \%$ of the maximal effect) of MaR1 and found that the values were $1.12 \mathrm{ng} / \mathrm{ml}$ and $0.99 \mathrm{ng} / \mathrm{ml}$ for promoting the neurite length and number, respectively (Figure 2E and 2F). Moreover, we compared the effects of NGF and MaR1 at the same dosage on neurite outgrowth. Interestingly, $10 \mathrm{ng} / \mathrm{ml} \mathrm{MaR} 1$ had a much stronger ability to increase neurite length than NGF at the same concentration (Figure $2 \mathrm{G}$ ), but their abilities to increase neurite numbers were not significantly different (Figure 2H). Taken together, our results suggest that MaR1 promotes neurite outgrowth in DRG cells cultured in vitro at a lower dosage than that required for NGF.

\section{MaR1 reduced DRG neuronal damage and promoted nerve path reconstruction after nerve injury}

Next, we examined whether MaR1 could attenuate DRG neuronal damage induced by nerve crush injury in mice. After the surgery, the damaged nerves of mice in the experimental group were covered with a sterilized hemostatic gelatin sponge containing $500 \mathrm{ng}$ of MaR1, while those of mice in the control group were covered with the saline vehicle. Immunohistochemical analysis of ATF3 showed that MaR1 significantly reduced the number of damaged DRG neurons after 7 days of treatment (Figure 3A and 3B). Our results suggested that MaR1 not only prohibited more DRG neuronal damage after nerve crush injury but also promoted the recovery of motor nerves and nerve path reconstruction. To confirm this hypothesis, we observed the regeneration of DRG neurons by intraplantarly injecting Dil, a fluorescent tracer dye, at 7 days after nerve injury. Two days later, more neurons were dyed with Dil in the MaR1 group than in the vehicle control group (Figure $3 \mathrm{C}$ and 3D), which indicated that MaR1 promoted nerve path reconstruction. Because muscle atrophy is a common phenomenon after PNI, we isolated the gastrocnemius muscles from mice treated with MaR1 or saline at 9 days after surgery to evaluate the ability of MaR1 to prevent muscle atrophy. Unsurprisingly, both the size and weight of the gastrocnemius muscles in the MaR1 group were larger than those of the vehicle group (Figure 3E and 3F). Taken 
together, these results showed that MaR1 reduced DRG neuronal damage, inhibited muscle atrophy, and promoted the regeneration of DRG neurons after nerve crush injury.

\section{MaR1 promoted functional recovery after nerve injury more effectively than NGF}

Because MaR1 administered at a lower dosage promoted neurite outgrowth better than NGF (Figure 2G and $2 \mathrm{H}$ ), we next compared the effects of MaR1 and NGF at the same dose (500 ng) on motor function and sensory function recovery in mice with nerve crush injury. Although the basic motor functions measured by the footprint test were not significantly different between the two groups of mice treated with MaR1 and NGF separately, the complex motor function measured by the rotarod test showed that MaR1 worked more quickly and efficiently than NGF to promote motor function recovery after nerve crushing (Figure $3 \mathrm{G}$ and $3 \mathrm{H}$ ). Similar results were obtained in the von Frey and Hargreaves tests, as MaR1 promoted the recovery of normal mechanical and thermal sensations after nerve injury faster than NGF (Figure $3 \mathrm{I}$ and $3 \mathrm{~J}$ ). Therefore, we suggest that MaR1 is better for PNI treatment than NGF, which requires a higher dosage and a longer treatment time.

\section{MaR1 alleviated nerve injury-induced neuropathic pain}

Next, we evaluated the effects of MaR1 on neuropathic pain in $\mathrm{CCl}$ mice. As shown in Figure 4A and 4B, the ipsilateral paw withdrawal threshold and paw withdrawal latency were dramatically decreased at 1 week after the surgery. The intrathecal injection of MaR1 (10 ng or $100 \mathrm{ng}$ ) rapidly reversed mechanical allodynia within $1 \mathrm{~h}$, and the effect lasted for more than $3 \mathrm{~h}$ and was dose-dependent (Figure 4A). Similarly, MaR1 also reduced the thermal hyperalgesia in $\mathrm{CCl}$ mice (Figure 4B). The analgesic effects of MaR1 disappeared at $24 \mathrm{~h}$ after treatment.

Transient receptor potential vanilloid 1 (TRPV1) is a type of TRP channel that plays an important role in mediating somatic inflammatory pain after injury [22, 23], and substantial efforts have been made to develop small-molecule inhibitors of TRP channels (e.g., TRPV1 and TRPA1). A previous study suggested that MaR1 inhibits capsaicin-induced TRPV1 currents and neuronal activity in trigeminal ganglion and DRG neurons $[12,24]$. Here, our patch-clamp recording results showed that $100 \mathrm{nM}$ capsaicin elicited a noticeable inward current in the perfusion of mouse DRG neurons, and the inward current was almost completely inhibited by pretreatment with MaR1 ( $3 \mathrm{ng} / \mathrm{ml}$ ) (Figure 4C), which is consistent with previous studies reporting that MaR1 potently modulates TRPV1.

\section{MaR1 inhibited glial cell activation and inflammation in the spinal cord after nerve crush injury}

Maintenance of the glial cell resting state is important for central nervous system homeostasis $[25,26]$. The activation of glial cells by noxious stimuli and inflammation triggers an inflammatory response, thereby inducing neuropathic pain. Therefore, we evaluated the expression of the astrocytic marker GFAP and the microglial marker IBA-1 in L4-L6 spinal dorsal horn sections at 7 days after sciatic nerve crush injury by double immunohistochemistry analysis. In the vehicle group, the fluorescence intensities of GFAP and IBA-1 immunoreactivity in the spinal cord dorsal horn were markedly upregulated on the 
ipsilateral side compared with the contralateral side (Figure 4D-F). In contrast, the intensities of GFAP and IBA-1 immunoreactivity on the ipsilateral side were significantly reduced in the group treated with the sterile gelatin sponge containing $500 \mathrm{ng}$ of MaR1 immediately after nerve crush injury (Figure 4D-F).

Due to their roles in inflammatory propagation and neutrophil recruitment, proinflammatory cytokines are important in the initiation and maintenance of neuropathic pain [27]. The expression levels of the inflammatory factors $I L 1 \beta$, ILG and TNF- $\square$ in the ipsilateral L4-L6 spinal dorsal horn were increased compared with those on the contralateral side at 7 days after the nerve crush injury as determined by qRTPCR (Figure 4G). However, the levels of these inflammatory factors were significantly suppressed by the local application of MaR1 to the injured nerve (Figure 4G). These results suggest that MaR1 attenuates nerve injury-induced neuropathic pain by repressing the astrocyte and microglia activation and proinflammatory cytokine production in the spinal cord.

\section{MaR1 promoted nerve growth via the PI3K-AKT-mTOR signaling pathway}

To assess the mechanisms underlying the analgesic effect of MaR1 in PNI, we examined its influence on the basic pain thresholds of naïve mice and compared it with that of NGF. After the intraplantar injection of saline (20 $\mu \mathrm{l})$, MaR1 (50 ng in $20 \mu \mathrm{l}$ saline) and NGF (50 ng in $20 \mu \mathrm{l}$ saline), we observed no significant changes in the pain thresholds between the vehicle and MaR1 groups (Figure 5A and 5B). However, the pain thresholds were decreased significantly in the NGF group at all the tested time points as determined by the von Frey and Hargreaves tests (Figure 5A and 5B). Thus, given the role of NGF in promoting pain, MaR1 is much better for the treatment of nerve injury.

Because the mechanisms underlying MaR1 function are complicated and relatively unknown, we investigated whether MaR1 functions via the same pathway as NGF to better understand how it regulates neuronal growth. We treated ND7/23 cells with MaR1 or NGF for 60 minutes, and western blot analysis revealed that phosphorylated AKT (p-AKT) was upregulated in the MaR1 and NGF groups compared with the vehicle group, while phosphorylated ERK ( $p$-ERK) was upregulated in only the NGF group compared with the vehicle group (Figure $5 \mathrm{C}$ and $5 \mathrm{D}$ ). There were no detectable differences in AKT and ERK expression among all three groups (Figure 5C and 5D). We next examined the effect of MaR1 on the phosphorylation of mTOR, a common downstream protein in the Akt signaling pathway. Phosphorylated mTOR (p-mTOR) levels were increased in both the MaR1 and NGF treatment groups, and the protein expression of mTOR was not significantly different among the three groups (Figure 5E and 5F). The PI3KAKT pathway is well known to mediate neuronal survival, and we next investigated the protein expression of phosphorylated PI3K (p-PI3K). Our results clearly demonstrated that MaR1 triggered p-PI3K protein expression, like NGF, did but did not affect PI3K expression (Figure 5E and 5F). Taken together, our results showed that MaR1 regulates neural growth through the PI3K-AKT-mTOR signaling pathway.

\section{Discussion}

PNI is a common occurrence that causes motor, sensory and autonomic nervous system dysfunction as well as neuropathic pain. While surgical intervention is the main treatment for $\mathrm{PNI}$, conservative and 
pharmacological treatments as well as cell-based therapies, gene therapies and growth factors are also popular for patients. Several growth factors have already been identified and preclinically applied for the treatment of PNI, and NGF is one of the most well studied. NGF plays vital roles in promoting the growth and survival of neurons. In recent decades, an increasing number of studies have suggested that NGF antagonism can ameliorate pain and pain-related behavior [3, 28-32]. Preclinical and clinical trials have demonstrated that the direct intradermal injection of NGF into rodents and humans clearly activates and sensitizes nociceptors [33-35]. Therefore, a variety of strategies have been designed to target the NGF pathway and thereby reduce neuropathic pain. MaR1, a newly identified anti-inflammatory and proresolving mediator, could be an important reagent for conservative treatment. Here, we showed that MaR1 stimulated the DRG growth much more strongly than NGF at the same dosage (Figure $2 \mathrm{G}$ and $2 \mathrm{H}$ ). We also demonstrated that MaR1 protected damaged DRG neurons and promoted functional neurological recovery. In mice with sciatic nerve crush injury, MaR1 at a low dose accelerated the recovery of both motor function and sensory function, promoted neural regeneration, and reduced DRG neuronal damage (Figure 3I-J), which suggests that the use of MaR1 for PNI treatment will ease the economic burdens on patients.

In addition, the paw withdrawal threshold and paw withdrawal latency were decreased after the injection of NGF into the plantar tissues of normal mice, while there were no changes in these parameters in the mice receiving the MaR1 injection (Figure 5A and B). This result suggests that even though NGF plays a neuroprotective role, its side effect of inducing mechanical and thermal pain represents a significant limitation. In contrast, MaR1 was not shown to induce pain but rather inhibited CCl-induced neuropathic pain development in our study (Figure $3 \mathrm{I}$ and $3 \mathrm{~J}$ ). The intrathecal injection of MaR1 into $\mathrm{CCl}$ model mice reduced allodynia to some extent in a dose-dependent manner (Figure $4 \mathrm{~A}$ and $4 \mathrm{~B}$ ). All of the above results strongly indicate that MaR1 should be considered a novel target for preclinical PNI treatment.

While the mechanisms of MaR1 are not well understood, those underlying NGF-induced pain have been studied extensively and might provide some clues about how MaR1 exerts its effects. Previous studies have demonstrated that NGF binds to TrkA-TrkA or TrkA-p75NTR to phosphorylate the TrkA cytoplasmic receptor and then triggers numerous second-messenger cascades (e.g., PI3K, AKT, ERK, mTOR) to affect the growth, differentiation and survival of neuronal cells [5, 36, 37]. AKT and ERK have been indicated to significantly contribute to the pathogenesis of various neurodegenerative diseases and to PNI [38, 39]. The PI3K/AKT pathway is activated by the NGF/TrkA complex and then participates in the cognitive dysfunctional pathological process [40], regulates neurotrophine retrograde axonal transportation in the nervous system [41] and determines neuronal polarity and axon growth [42, 43]. In neuronal cell lines, PI3K promotes the outgrowth and retraction of neurites [44, 45]. Since NGF is known to bind TrkA to initiate downstream signaling pathways, such as PI3K-AKT and Ras-MEK, followed by activation of the ERK or mTOR signal transduction pathway and, finally, the regulation of cytokine secretion, we aimed to examine whether MaR1 functions via a similar pathway in ND7/23 cells by western blot. The protein expression of p-AKT was improved to similar levels in the MaR1 and NGF treatment groups, while the AKT expression was not noticeably altered in either group (Figure 5C and 5D). However, neither the p-ERK level nor the ERK level was changed by the administration of MaR1, unlike in the NGF treatment group 
(Figure 5E and 5F). The expression levels of AKT downstream of p-mTOR and AKT upstream of PI3K were elevated in both the MaR1 and NGF treatment groups, which indicated that like NGF, MaR1 promotes the neuronal growth process via the PI3K-AKT-mTOR pathway, while NGF also functions via the PI3K-ERK pathway.

Thus far, little is known about the MaR1 receptors. Colas et al. discovered that MaR1 is a partial agonist of recombinant human leukotriene B4 receptor (BLT1), suggesting that BLT1 is a potential receptor for MaR1 [46]. Recently, human leucine-rich repeat containing G protein-coupled receptor 6 (LGR6), a plasma membrane GPCR, was screened out as a receptor for MaR1 [47]. MaR1 and LGR6 interactions in phagocytes were clearly demonstrated to play a role in resolving inflammation. Another confirmed receptor for MaR1 is retinoic acid-related orphan receptor a (RORa), which induces nonalcoholic steatohepatitis (NASH) protection through the MaR1/RORa/12-lipoxygenase (12-LOX) autoregulatory circuit (Han et al., 2019). RORa and LGR6 are the molecular targets for MaR1 in chronic NASH and acute sepsis, respectively. However, whether RORa or LGR6, or even both, functions as an MaR1 receptor and thereby triggers intracellular cascades to promote PNI recovery requires further study. A deeper and updated understanding of this phenomenon will likely significantly advance the chances of MaR1 being investigated in clinical trials.

In conclusion, we provided clear evidence that MaR1 should be considered a novel analgesic agent for the treatment of neuropathic pain and as a new activator for nerve regeneration to improve functional neurological recovery after nerve crush injury. MaR1 should be considered as the mainstay treatment for PNI rather than NGF, which is expensive and has some uncertain adverse effects.

\section{Conclusions}

Peripheral nerve injury (PNI) is a public health concern that results in sensory and motor disorders as well as neuropathic pain and secondary lesions. Currently, effective treatments for PNI are still limited. One well-known treatment is nerve growth factor (NGF), which improves nerve regeneration but accelerates neuropathic pain. New cytokine(s) or drug molecular(s) that function better than NGF are urgently needed for patients with PNI. Maresin 1 (MaR1) is an anti-inflammatory and proresolving mediator that has the potential to regenerate tissue. Our study demonstrated that the administration of MaR1 to a mouse model of PNI not only promoted neurological functional recovery by protecting damaged DRG neurons and promoting injured axonal regeneration but also alleviated neuropathic pain by inhibiting glial cell activation and the inflammatory response in the spinal cord. Therefore, our results suggest that MaR1 is a promising therapeutic agent for PNI.

\section{List Of Abbreviations}

PNI: peripheral nerve injury

NGF: nerve growth factor 
MaR1: Maresin 1

TRPV1: transient receptor potential vanilloid 1

DRG: dorsal root ganglion

CCl: chronic constriction injury model

SFI: sciatic function index

TNF-a: tumor necrosis factor-alpha

IL-1 $\beta$ : interleukin (IL)-1-beta

IL-6: interleukin (IL)-6

ATF-3: activating Transcription Factor 3

GFAP: glial fibrillary acidic protein

IBA1: ionized calcium binding adapter molecule 1

NF200: neurofilament 200

p-AKT: phosphorylated AKT

p-ERK: phosphorylated ERK

p-PI3K: phosphorylated PI3K

p-mTOR: phosphorylated mTOR

BLT1: leukotriene B4 receptor

LGR6: leucine-rich repeat containing G protein-coupled receptor 6

RORa: retinoic acid-related orphan receptor a

NASH: nonalcoholic steatohepatitis

12-LOX: 12-lipoxygenase

\section{Declarations}

\section{Ethics approval and consent to participate}

Mouse experiments were performed in accordance with the International Guidelines for animal research. 
All procedures involving animals were approved according to guidelines established by the Institutional Animal Care and Use Committee of Nantong University.

\section{Consent for publication}

Not applicable.

\section{Availability of data and materials}

The datasets used and/or analyzed during the current study are available from the corresponding author on reasonable request.

\section{Competing interests}

The authors declare no competing financial interests.

\section{Funding}

This work was supported by the National Natural Science Foundation of China (31872773, 32070998), the Key Research and Development Program (Social Development) of Jiangsu Province (BE2020667), the Foundation of Jiangsu Province "333 Project High-level Talents" (BRA2020076), Six Talent Peaks project in Jiangsu Province (2017-SWYY-056), China Postdoctoral Science Foundation funded project (2020M670042ZX), Nantong Science and Technology Bureau (MS12020056), and Priority Academic Program Development of Jiangsu Higher Education Institutions (PAPD).

\section{Authors' contributions}

G.C. and Y.C. designed the experiments; J.H.W., W.F.S., Y.Y.Z., Z.Y.W., Y.C.H. P.X. and X.Z. performed the experiments; G.C. and J.H.W. drafted the manuscript. All authors contributed to the manuscript revision, read, and approved the submitted version.

\section{Acknowledgements}

Not applicable.

\section{References}

1. Faroni A, Mobasseri SA, Kingham PJ, Reid AJ. Peripheral nerve regeneration: experimental strategies and future perspectives. Advanced drug delivery reviews. 2015;82-83:160-7; doi: 10.1016/j.addr.2014.11.010.

2. Gu X, Ding F, Williams DF. Neural tissue engineering options for peripheral nerve regeneration. Biomaterials. 2014;35 24:6143-56; doi: 10.1016/j.biomaterials.2014.04.064.

3. McMahon SB, Bennett DL, Priestley JV, Shelton DL. The biological effects of endogenous nerve growth factor on adult sensory neurons revealed by a trkA-lgG fusion molecule. Nature medicine. 
1995;1 8:774-80; doi: 10.1038/nm0895-774.

4. Siniscalco D, Giordano C, Rossi F, Maione S, de Novellis V. Role of neurotrophins in neuropathic pain. Current neuropharmacology. 2011;9 4:523-9; doi: 10.2174/157015911798376208.

5. Wise BL, Seidel MF, Lane NE. The evolution of nerve growth factor inhibition in clinical medicine. Nature reviews Rheumatology. 2021;17 1:34-46; doi: 10.1038/s41584-020-00528-4.

6. Serhan CN, Yang R, Martinod K, Kasuga K, Pillai PS, Porter TF, et al. Maresins: novel macrophage mediators with potent antiinflammatory and proresolving actions. The Journal of experimental medicine. 2009;206 1:15-23; doi: 10.1084/jem.20081880.

7. Serhan CN, Dalli J, Colas RA, Winkler JW, Chiang N. Protectins and maresins: New pro-resolving families of mediators in acute inflammation and resolution bioactive metabolome. Biochimica et biophysica acta. 2015;1851 4:397-413; doi: 10.1016/j.bbalip.2014.08.006.

8. Krishnamoorthy N, Burkett PR, Dalli J, Abdulnour RE, Colas R, Ramon S, et al. Cutting edge: maresin-1 engages regulatory $T$ cells to limit type 2 innate lymphoid cell activation and promote resolution of lung inflammation. Journal of immunology (Baltimore, Md : 1950). 2015;194 3:863-7; doi: 10.4049/jimmunol.1402534.

9. Wang YH, Li Y, Wang JN, Zhao QX, Jin J, Wen S, et al. Maresin 1 Attenuates Radicular Pain Through the Inhibition of NLRP3 Inflammasome-Induced Pyroptosis via NF-kB Signaling. Frontiers in neuroscience. 2020;14:831; doi: 10.3389/fnins.2020.00831.

10. Francos-Quijorna I, Santos-Nogueira E, Gronert K, Sullivan AB, Kopp MA, Brommer B, et al. Maresin 1 Promotes Inflammatory Resolution, Neuroprotection, and Functional Neurological Recovery After Spinal Cord Injury. The Journal of neuroscience : the official journal of the Society for Neuroscience. 2017;37 48:11731-43; doi: 10.1523/jneurosci.1395-17.2017.

11. Xian W, Wu Y, Xiong W, Li L, Li T, Pan S, et al. The pro-resolving lipid mediator Maresin 1 protects against cerebral ischemia/reperfusion injury by attenuating the pro-inflammatory response. Biochemical and biophysical research communications. 2016;472 1:175-81; doi: 10.1016/j.bbrc.2016.02.090.

12. Serhan CN, Dalli J, Karamnov S, Choi A, Park CK, Xu ZZ, et al. Macrophage proresolving mediator maresin 1 stimulates tissue regeneration and controls pain. FASEB journal : official publication of the Federation of American Societies for Experimental Biology. 2012;26 4:1755-65; doi: 10.1096/fj.11201442.

13. Wang CW, Yu SH, Fretwurst T, Larsson L, Sugai JV, Oh J, et al. Maresin 1 Promotes Wound Healing and Socket Bone Regeneration for Alveolar Ridge Preservation. Journal of dental research. 2020;99 8:930-7; doi: 10.1177/0022034520917903.

14. Huang R, Vi L, Zong X, Baht GS. Maresin 1 resolves aged-associated macrophage inflammation to improve bone regeneration. FASEB journal : official publication of the Federation of American Societies for Experimental Biology. 2020;34 10:13521-32; doi: 10.1096/fj.202001145R.

15. Su WF, Wu F, Jin ZH, Gu Y, Chen YT, Fei Y, et al. Overexpression of P2X4 receptor in Schwann cells promotes motor and sensory functional recovery and remyelination via BDNF secretion after nerve 
injury. Glia. 2019;67 1:78-90; doi: 10.1002/glia.23527.

16. Chen G, Luo X, Qadri MY, Berta T, Ji RR. Sex-Dependent Glial Signaling in Pathological Pain: Distinct Roles of Spinal Microglia and Astrocytes. Neuroscience bulletin. 2018;34 1:98-108; doi: 10.1007/s12264-017-0145-y.

17. Su WF, Gu Y, Wei ZY, Shen YT, Jin ZH, Yuan Y, et al. Rab27a/SIp2-a complex is involved in Schwann cell myelination. Neural Regen Res. 2016;11 11:1830-8; doi: 10.4103/1673-5374.194755.

18. Chen G, Kim YH, Li H, Luo H, Liu DL, Zhang ZJ, et al. PD-L1 inhibits acute and chronic pain by suppressing nociceptive neuron activity via PD-1. Nature neuroscience. 2017;20 7:917-26; doi: $10.1038 / \mathrm{nn} .4571$.

19. Chen G, Park CK, Xie RG, Ji RR. Intrathecal bone marrow stromal cells inhibit neuropathic pain via TGF-beta secretion. The Journal of clinical investigation. 2015;125 8:3226-40; doi: $10.1172 / \mathrm{JCl} 80883$.

20. Yang T, Xing L, Yu W, Cai Y, Cui S, Chen G. Astrocytic reprogramming combined with rehabilitation strategy improves recovery from spinal cord injury. FASEB journal : official publication of the Federation of American Societies for Experimental Biology. 2020;34 11:15504-15; doi: 10.1096/fj.202001657RR.

21. Lackington WA, Kočí Z, Alekseeva T, Hibbitts AJ, Kneafsey SL, Chen G, et al. Controlling the dosedependent, synergistic and temporal effects of NGF and GDNF by encapsulation in PLGA microparticles for use in nerve guidance conduits for the repair of large peripheral nerve defects. Journal of controlled release : official journal of the Controlled Release Society. 2019;304:51-64; doi: 10.1016/j.jconrel.2019.05.001.

22. Bautista DM, Jordt SE, Nikai T, Tsuruda PR, Read AJ, Poblete J, et al. TRPA1 mediates the inflammatory actions of environmental irritants and proalgesic agents. Cell. 2006;124 6:1269-82; doi: 10.1016/j.cell.2006.02.023.

23. Kosugi M, Nakatsuka T, Fujita T, Kuroda Y, Kumamoto E. Activation of TRPA1 channel facilitates excitatory synaptic transmission in substantia gelatinosa neurons of the adult rat spinal cord. The Journal of neuroscience : the official journal of the Society for Neuroscience. 2007;27 16:4443-51; doi: 10.1523/jneurosci.0557-07.2007.

24. Park CK. Maresin 1 Inhibits TRPV1 in Temporomandibular Joint-Related Trigeminal Nociceptive Neurons and TMJ Inflammation-Induced Synaptic Plasticity in the Trigeminal Nucleus. Mediators of inflammation. 2015;2015:275126; doi: 10.1155/2015/275126.

25. Wang $D$, Couture R, Hong Y. Activated microglia in the spinal cord underlies diabetic neuropathic pain. European journal of pharmacology. 2014;728:59-66; doi: 10.1016/j.ejphar.2014.01.057.

26. Jha MK, Jeon S, Suk K. Glia as a Link between Neuroinflammation and Neuropathic Pain. Immune network. 2012;12 2:41-7; doi: 10.4110/in.2012.12.2.41.

27. Denes A, Thornton P, Rothwell NJ, Allan SM. Inflammation and brain injury: acute cerebral ischaemia, peripheral and central inflammation. Brain, behavior, and immunity. 2010;24 5:708-23; doi: 10.1016/j.bbi.2009.09.010. 
28. Lewin GR, Rueff A, Mendell LM. Peripheral and central mechanisms of NGF-induced hyperalgesia. The European journal of neuroscience. 1994;6 12:1903-12; doi: 10.1111/j.14609568.1994.tb00581.x.

29. Woolf CJ, Safieh-Garabedian B, Ma QP, Crilly P, Winter J. Nerve growth factor contributes to the generation of inflammatory sensory hypersensitivity. Neuroscience. 1994;62 2:327-31; doi: 10.1016/0306-4522(94)90366-2.

30. Sevcik MA, Ghilardi JR, Peters CM, Lindsay TH, Halvorson KG, Jonas BM, et al. Anti-NGF therapy profoundly reduces bone cancer pain and the accompanying increase in markers of peripheral and central sensitization. Pain. 2005;115 1-2:128-41; doi: 10.1016/j.pain.2005.02.022.

31. Sabsovich I, Wei T, Guo TZ, Zhao R, Shi X, Li X, et al. Effect of anti-NGF antibodies in a rat tibia fracture model of complex regional pain syndrome type I. Pain. 2008;138 1:47-60; doi: 10.1016/j.pain.2007.11.004.

32. Zahn PK, Subieta A, Park SS, Brennan TJ. Effect of blockade of nerve growth factor and tumor necrosis factor on pain behaviors after plantar incision. The journal of pain : official journal of the American Pain Society. 2004;5 3:157-63; doi: 10.1016/j.jpain.2004.02.538.

33. Andreev N, Dimitrieva N, Koltzenburg M, McMahon SB. Peripheral administration of nerve growth factor in the adult rat produces a thermal hyperalgesia that requires the presence of sympathetic post-ganglionic neurones. Pain. 1995;63 1:109-15; doi: 10.1016/0304-3959(95)00024-m.

34. Dyck PJ, Peroutka S, Rask C, Burton E, Baker MK, Lehman KA, et al. Intradermal recombinant human nerve growth factor induces pressure allodynia and lowered heat-pain threshold in humans. Neurology. 1997;48 2:501-5; doi: 10.1212/wnl.48.2.501.

35. Mantyh PW, Koltzenburg M, Mendell LM, Tive L, Shelton DL. Antagonism of nerve growth factor-TrkA signaling and the relief of pain. Anesthesiology. 2011;115 1:189-204; doi: 10.1097/ALN.0b013e31821b1ac5.

36. Bibel M, Hoppe E, Barde YA. Biochemical and functional interactions between the neurotrophin receptors trk and p75NTR. The EMBO journal. 1999;18 3:616-22; doi: 10.1093/emboj/18.3.616.

37. Greene LA, Kaplan DR. Early events in neurotrophin signalling via Trk and p75 receptors. Current opinion in neurobiology. 1995;5 5:579-87; doi: 10.1016/0959-4388(95)80062-x.

38. Rai SN, Dilnashin H, Birla H, Singh SS, Zahra W, Rathore AS, et al. The Role of PI3K/Akt and ERK in Neurodegenerative Disorders. Neurotoxicity research. 2019;35 3:775-95; doi: 10.1007/s12640-0190003-y.

39. Huang HT, Sun ZG, Liu HW, Ma JT, Hu M. ERK/MAPK and PI3K/AKT signal channels simultaneously activated in nerve cell and axon after facial nerve injury. Saudi journal of biological sciences. 2017;24 8:1853-8; doi: 10.1016/j.sjbs.2017.11.027.

40. Wang $X$, Zhao L. Calycosin ameliorates diabetes-induced cognitive impairments in rats by reducing oxidative stress via the PI3K/Akt/GSK-3 $\beta$ signaling pathway. Biochemical and biophysical research communications. 2016;473 2:428-34; doi: 10.1016/j.bbrc.2016.03.024. 
41. Cai F, Helke CJ. Abnormal PI3 kinase/Akt signal pathway in vagal afferent neurons and vagus nerve of streptozotocin-diabetic rats. Brain research Molecular brain research. 2003;110 2:234-44; doi: 10.1016/s0169-328x(02)00652-6.

42. Jiang H, Guo W, Liang X, Rao Y. Both the establishment and the maintenance of neuronal polarity require active mechanisms: critical roles of GSK-3beta and its upstream regulators. Cell. 2005;120 1:123-35; doi: 10.1016/j.cell.2004.12.033.

43. Shi SH, Jan LY, Jan YN. Hippocampal neuronal polarity specified by spatially localized mPar3/mPar6 and PI 3-kinase activity. Cell. 2003;112 1:63-75; doi: 10.1016/s0092-8674(02)01249-7.

44. Rodgers EE, Theibert AB. Functions of PI 3-kinase in development of the nervous system. International journal of developmental neuroscience : the official journal of the International Society for Developmental Neuroscience. 2002;20 3-5:187-97; doi: 10.1016/s0736-5748(02)00047-3.

45. Leemhuis J, Boutillier S, Barth H, Feuerstein TJ, Brock C, Nürnberg B, et al. Rho GTPases and phosphoinositide 3-kinase organize formation of branched dendrites. The Journal of biological chemistry. 2004;279 1:585-96; doi: 10.1074/jbc.M307066200.

46. Colas RA, Dalli J, Chiang N, Vlasakov I, Sanger JM, Riley IR, et al. Identification and Actions of the Maresin 1 Metabolome in Infectious Inflammation. Journal of immunology. 2016;197 11:4444-52; doi: $10.4049 /$ jimmunol.1600837.

47. Chiang N, Libreros S, Norris PC, de la Rosa X, Serhan CN. Maresin 1 activates LGR6 receptor promoting phagocyte immunoresolvent functions. The Journal of clinical investigation. 2019;129 12:5294-311; doi: 10.1172/jci129448.

\section{Figures}


A

Motor function: Footprint test

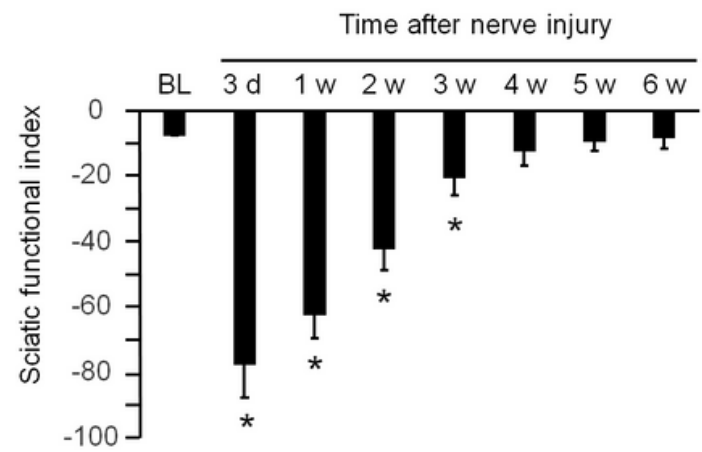

C Sensory function: von Frey test

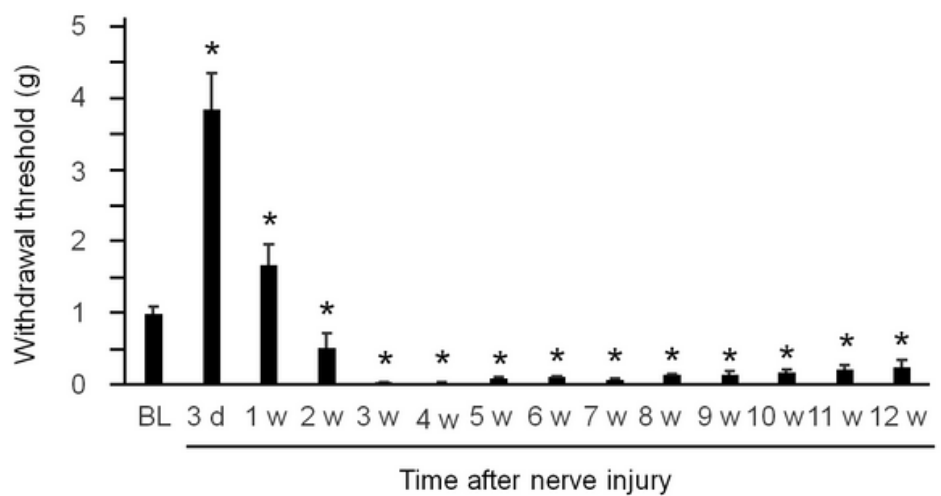

Motor function: Rotarod test

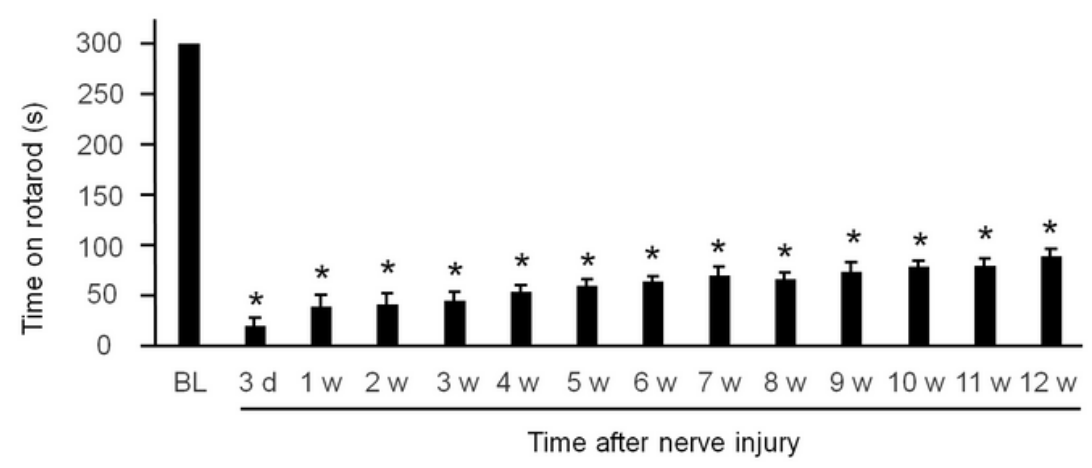

D Sensory function: Hargreaves test

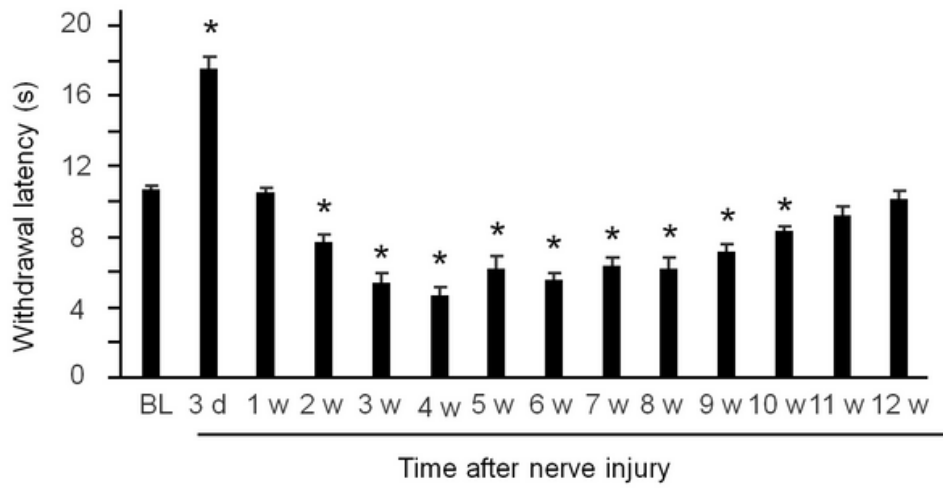

Figure 1

Motor and sensory dysfunction and neuropathic pain in mice with sciatic nerve crush injury. The motor functions of mice with sciatic nerve crush injury were measured by footprint (A) and rotarod (B) tests. The sensory functions of mice with sciatic nerve crush injury were measured by the von Frey (C) and Hargreaves (D) tests. The data are presented as the mean \pm SEM, $n=8$ mice in each group, ${ }^{*} p<0.05$ versus the baseline $(\mathrm{BL})$ group. 
A

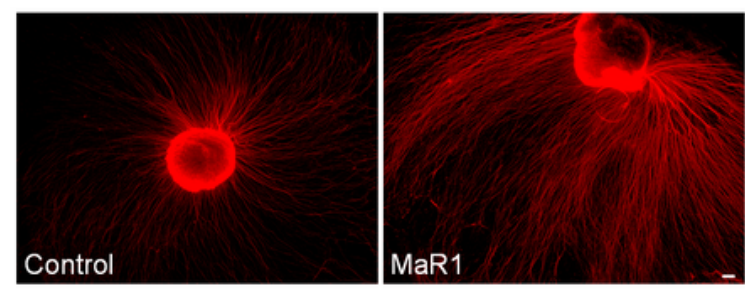

C

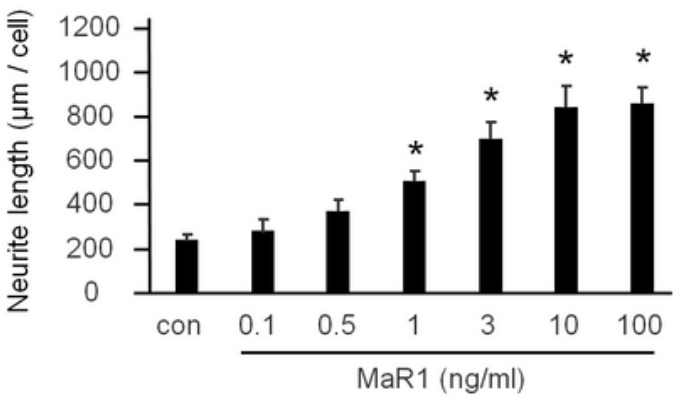

E

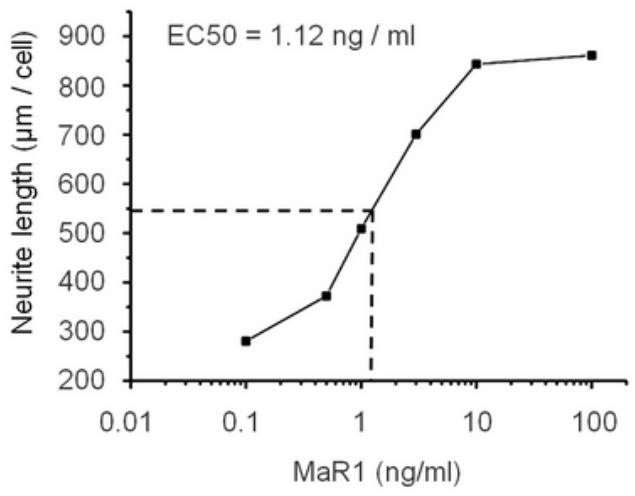

G

- MaR1 (ng/ml)

- NGF (ng/ml)

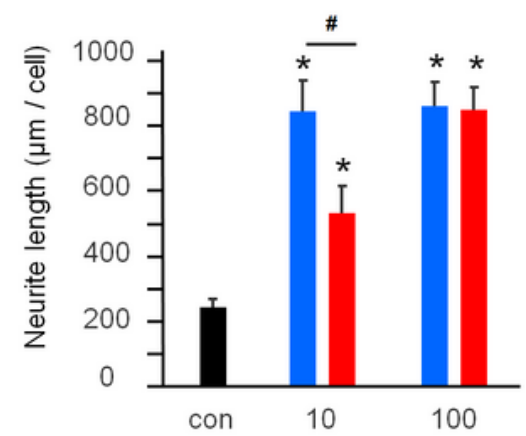

$\mathrm{H}$
B

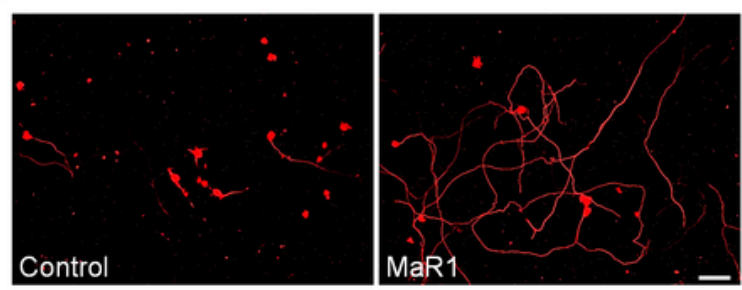

D

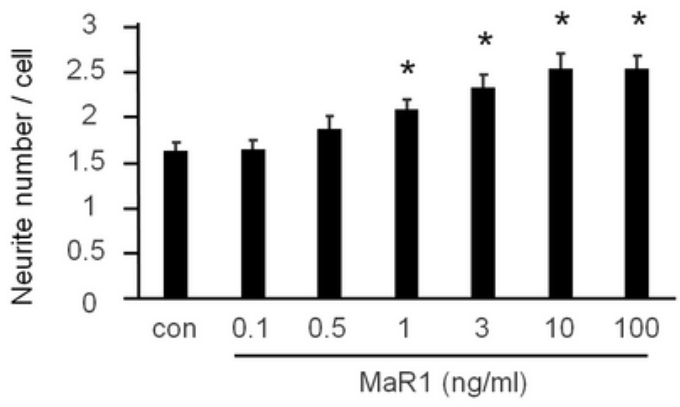

$\mathrm{F}$

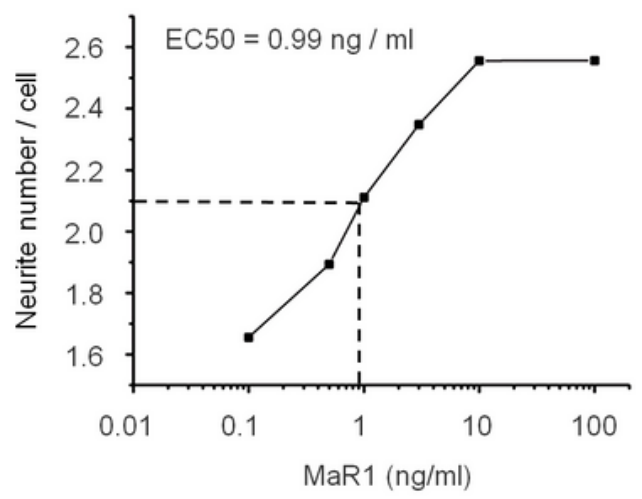

- MaR1 (ng/ml)

- NGF (ng/ml)

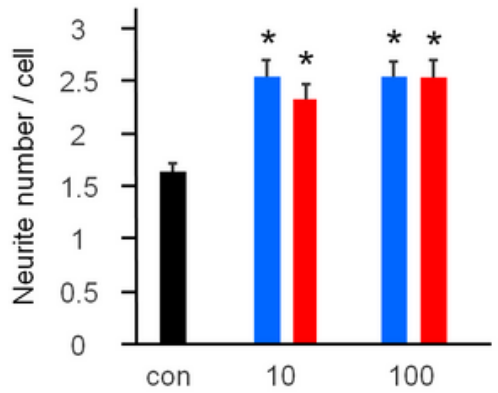

\section{Figure 2}

MaR1 dose-dependently promoted axon regeneration in vitro. MaR1 stimulated neurite outgrowth in DRG neurons grown in explant and dissociated cultures in vitro. Neurofilament staining was employed to examine the outgrowth of neurites in the DRG. (A) DRG neurons grown in explant cultures for 5 days and treated with (right) and without (left) MaR1. Scale bars $=100 \mu \mathrm{m}$. (B) DRG neurons grown in dissociated cultures for $36 \mathrm{~h}$ and treated with (right) and without (left) MaR1. Scale bars $=100 \mu \mathrm{m}$. (C, D) MaR1 
increased the neurite length and number in a dose-dependent manner. DRG neurons cultured with MaR1 for 36 h. $(E, F)$ The EC50 values at which MaR1 increased the neurite length and number were measured. $(G, H)$ Comparison of the effects of MaR1 and NGF at the same concentration on increasing the neurite length and number. The data are presented as the mean $\pm S E M,{ }^{*} p<0.05$ versus the control group; $\# p<0.05$ versus the NGF group.

A

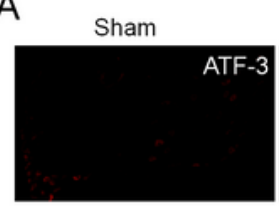

C Sham

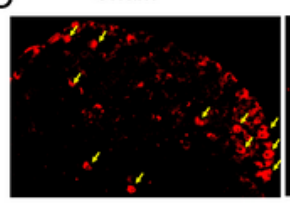

E

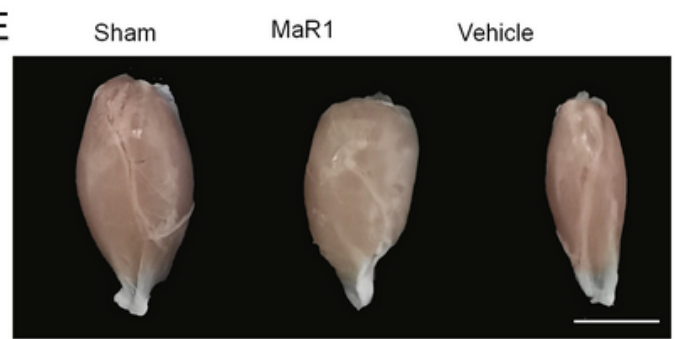

G

Motor function: Footprint test

"Vehicle MaR1 "NGF

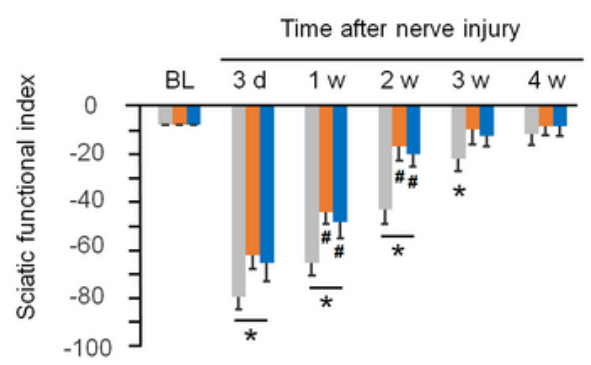

I

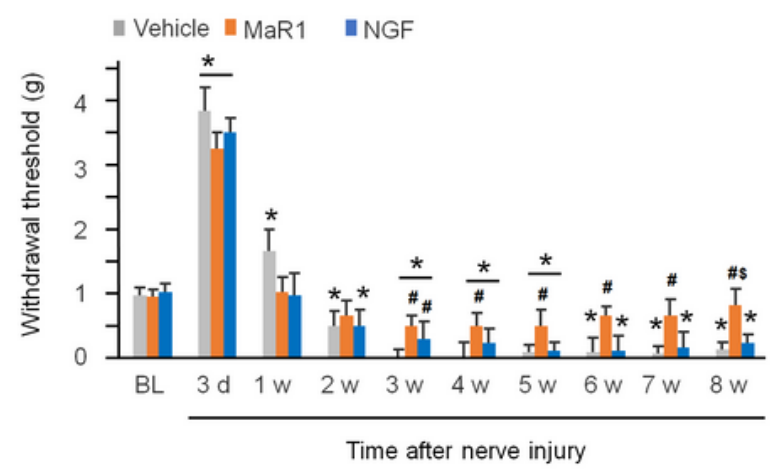

$\mathrm{H}$
B

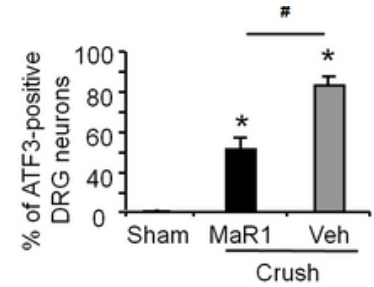

D

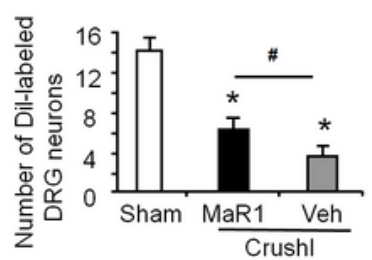

F

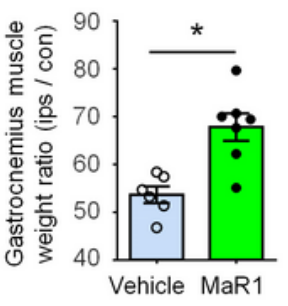

Motor function: Rotarod test " Vehicle " MaR1 "NGF

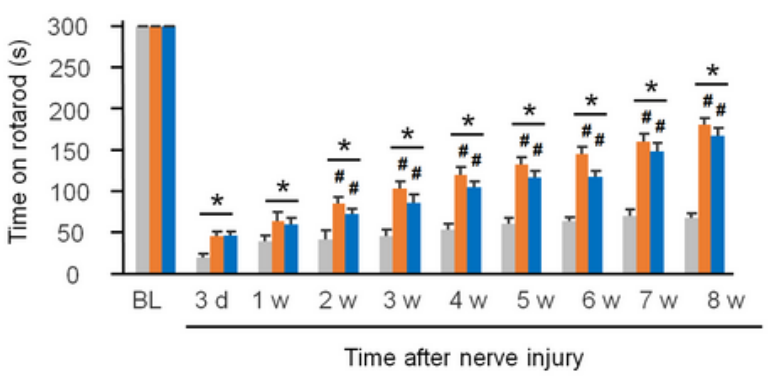

$\mathrm{J}$

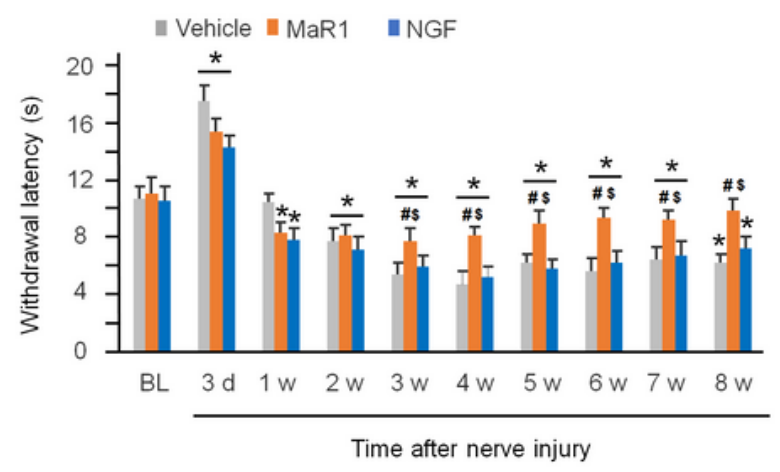

Figure 3 
MaR1 protected against DRG neuronal damage and improved functional neurological recovery. Immunohistochemical analysis of ATF3 was used to determine the number of DRG neurons $(A, B)$. Neuronal regeneration was detected by the Dil labeling of DRG neurons (C, D). MaR1 (500 ng) was administered to the injured region, scale bars $=50 \mu \mathrm{m}$. The data are presented as the mean \pm SEM, ${ }^{*} p<0.05$ versus the sham group; $\# p<0.05$ versus the vehicle group. $(E, F)$ The size and weight of the gastrocnemius muscle were measured. Scale bars $=5 \mathrm{~mm}$. The data are presented as the mean $\pm \mathrm{SEM}$, ${ }^{*} \mathrm{p}<0.05$ versus the vehicle group. (G-J) The local application of MaR1 (500 ng in a sterile gelatin sponge) or NGF (500 ng in sterile gelatin sponge) to the injured nerve promoted motor and sensory function recovery, but MaR 1 was more effective. The data are presented as the mean $\pm S E M, n=6$ mice in each group, ${ }^{*}<0.05$ versus the $B L$ group, $\# p<0.05$ versus the NGF group, $\$ p<0.05$ versus both the $B L$ and NGF groups. 
A

Sensory function: von Frey test

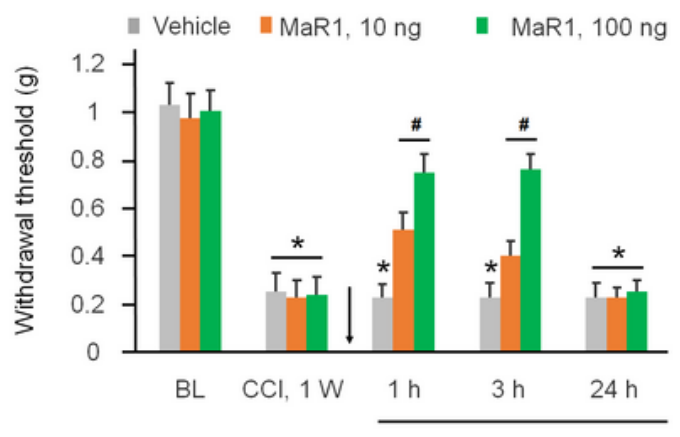

Time after intrathecal injection

C

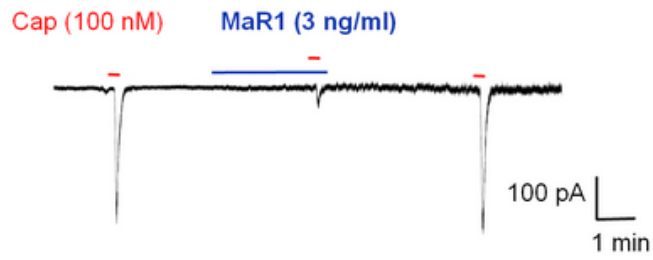

D
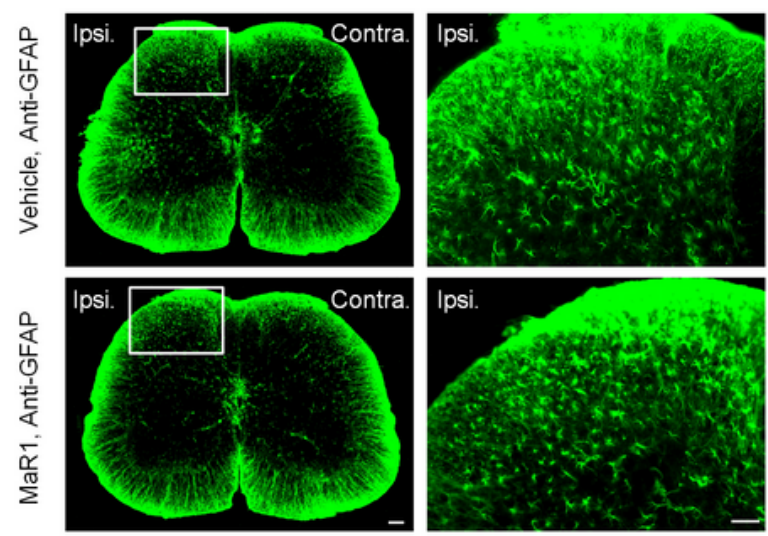

$E$
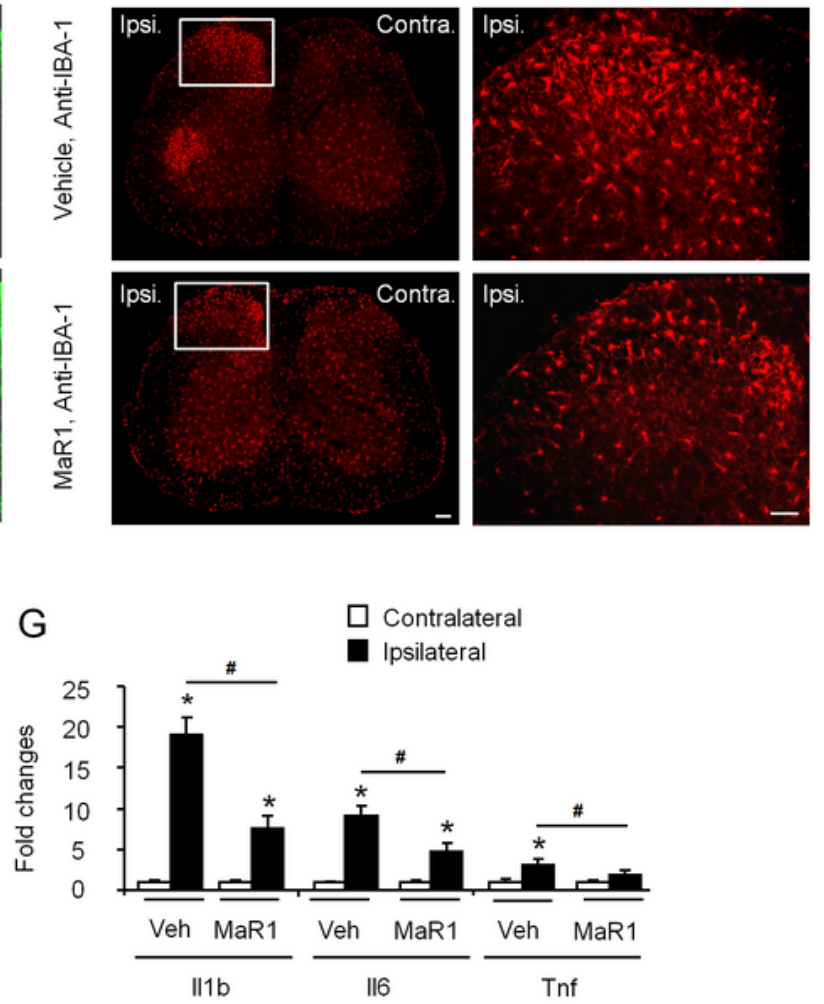

\section{Figure 4}

Maresin 1 alleviated nerve injury-induced neuropathic pain. Intrathecal injection of MaR1 attenuated the mechanical allodynia (A) and thermal hyperalgesia (B) induced by SCl. The data are presented as the mean $\pm S E M, n=6$ mice in each group, ${ }^{*} p<0.05$ versus the $B L$ group; $\# p<0.05$ versus the $10 \mathrm{ng}$ MaR 1 group. (C) MaR1 inhibited capsaicin (CAP; $100 \mathrm{nM}$ )-induced inward currents. The data are presented as the mean $\pm S E M, n=9$ neurons, ${ }^{\star} p<0.05$ versus the control group. (D, E, F) MaR1 suppressed the 
activation of astrocytes (immunofluorescence staining of GFAP) and microglia (immunofluorescence staining of IBA-1) in the L4-L6 spinal dorsal horn. ${ }^{*} p<0.05$ versus the contralateral group; $\# p<0.05$ versus the vehicle group. (G) qRT-PCR indicated that MaR1 inhibited the increased mRNA expression of $\|1 \beta\|$,6 , and TNF-a in the ipsilateral L4-L6 region induced by sciatic nerve crush injury. The data are presented as the mean $\pm S E M, n=4$ mice in each group. ${ }^{*} p<0.05$ versus the contralateral group; $\# p<0.05$ versus the vehicle group.

A

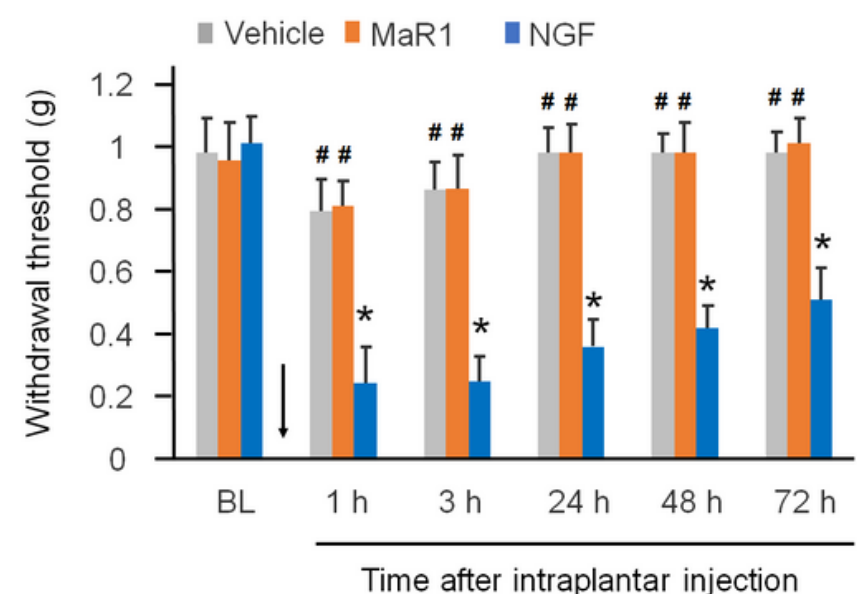

B

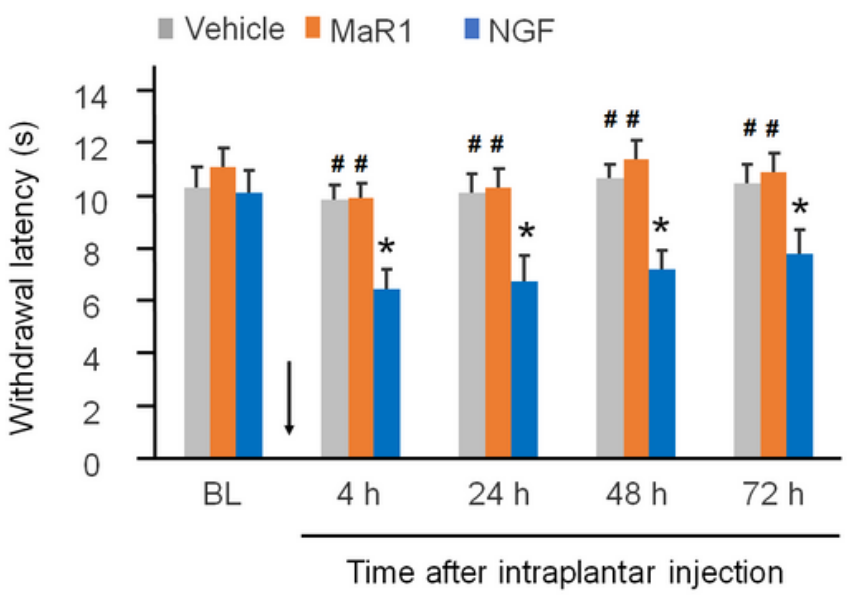

C

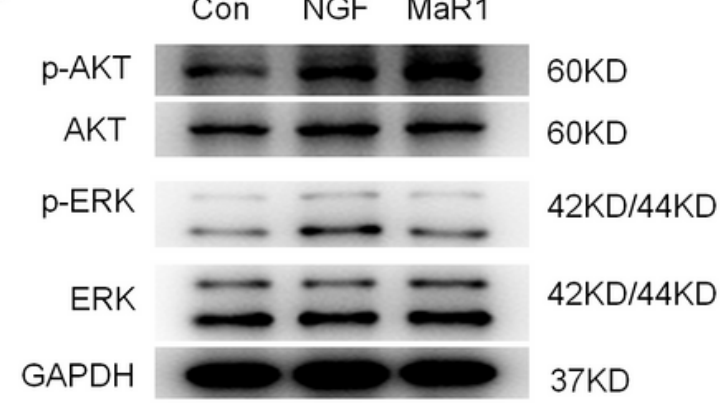

E

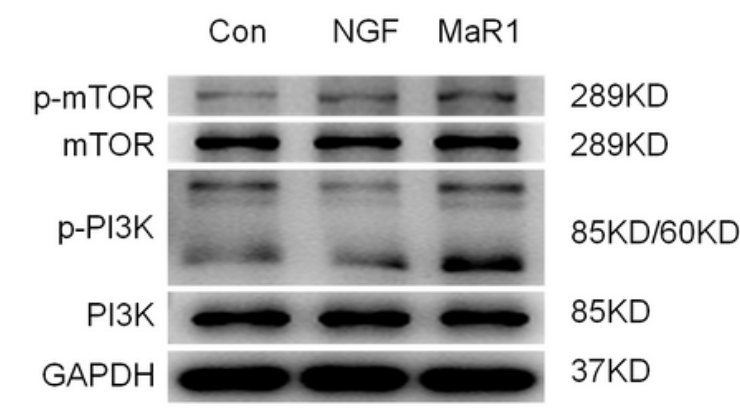

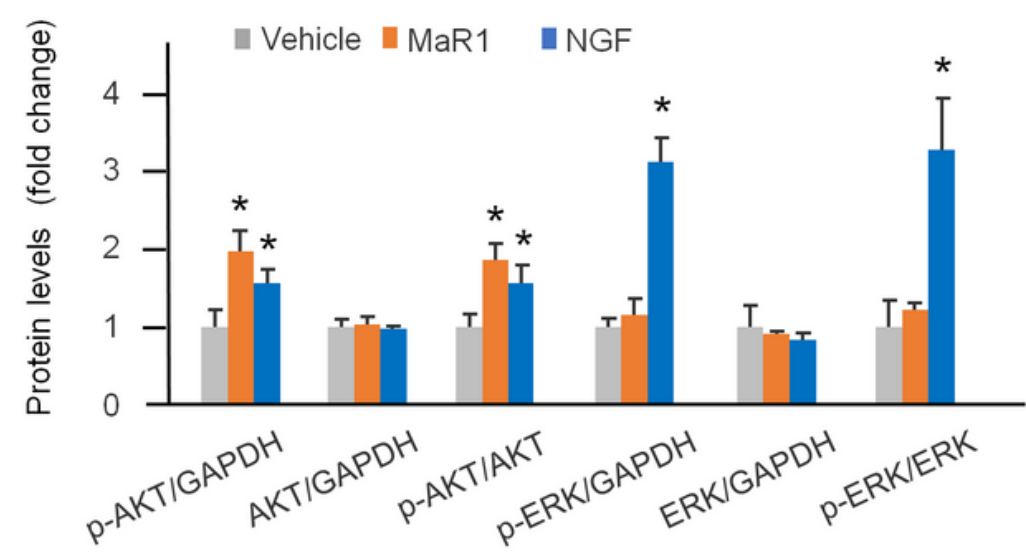

$\mathrm{F}$

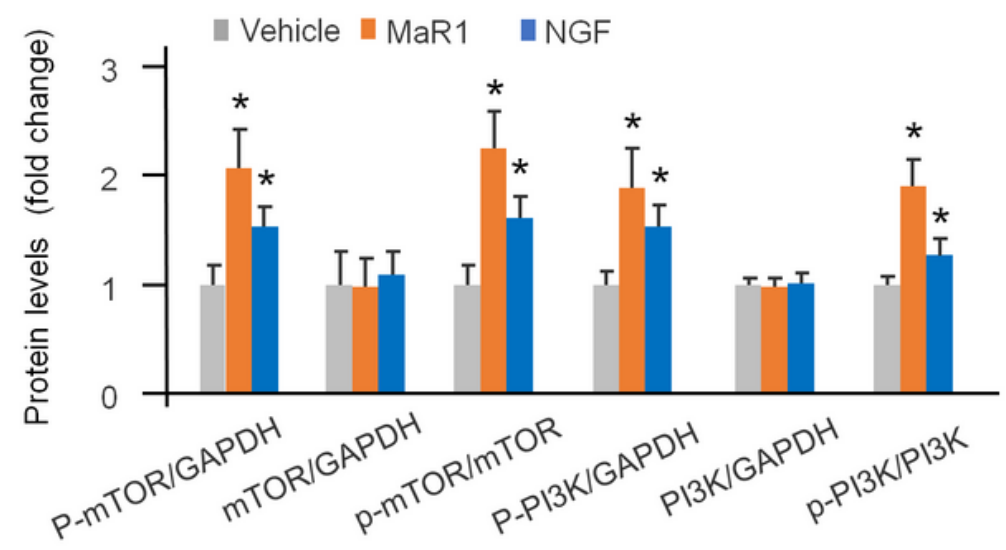

Figure 5 
Maresin 1 regulated the PI3K-AKT-mTOR pathway. (A, B) Intraplantar injection of MaR1 did not affect the mechanical withdrawal threshold or paw withdrawal latency of normal mice, while NGF did. The data are presented as the mean \pm SEM, $n=6$ mice in each group. ${ }^{*} p<0.05$ MaR1 group verse the vehicle group; $\# p<0.05$ NGF group verse the vehicle group. (C-F) Western blot analysis indicated that the administration of MaR1 to ND7/23 cells significantly activated the PI3K-AKT-mTOR pathway, while NGF promoted the expression of $\mathrm{p}-\mathrm{PI} 3 \mathrm{~K}, \mathrm{p}-\mathrm{AKT}$, $\mathrm{p}-\mathrm{mTOR}$ and $\mathrm{p}$-ERK. 